\title{
LncRNA SNHG5 promotes the proliferation and cancer stem cell-like properties of hepatocellular carcinoma by regulating UPF1 and Wnt signaling pathway
}

\section{Yarui Li}

The First Affiliated Hospital of Xi'an Jiaotong University https://orcid.org/0000-0001-9371-7622 Junbi Hu

The First Affiliated Hospital of Xi'an Jiaotong University

Guifang Lu

The First Affiliated Hospital of Xi'an Jiaotong University

\section{Mudan Ren}

The First Affiliated Hospital of Xi'an Jiaotong University

\section{Zhiyong Zhang}

The First Affiliated Hospital of Xi'an Jiaotong University

\section{Dan Guo}

The First Affiliated Hospital of Xi'an Jiaotong University

\section{Xinlan Lu}

The First Affiliated Hospital of Xi'an Jiaotong University

Shuixiang He ( $\nabla$ hesx321@sina.com )

\section{Research}

Keywords: SNHG5, UPF1, Liver CSCs, Wnt/ß-catenin , HCC

Posted Date: February 19th, 2020

DOI: https://doi.org/10.21203/rs.2.23923/v1

License: (c) (1) This work is licensed under a Creative Commons Attribution 4.0 International License. Read Full License 


\section{Abstract}

Background: It have been revealed that IncRNAs had a critical role in human cancers, including HCC. Our previous study found a IncRNA SNHG5 related to the development of HCC. However, the role and exact mechanism of SNHG5 in HCC proliferation and the liver CSC-like properties is still unclear.

Materials and Methods: We investigated the HCC cells proliferation and CSC-like properties in vitro and in vivo by knockdown or upregulate SNHG5, and detected underlying mechanisms by qRT-PCR, Western blotting, bioinformatic analysis, luciferase reporter assay, RNA immunoprecipitation.

Results: Knockdown of SNHG5 repressed HCC cells proliferation and CSC-like properties, while overexpression of SNHG5 promoted cells growth. At the same time, CSCs markers ( CD44, CD133 and ALDH1) and related transcription factors (OCT4, SOX2 and NANOG) were downregulated when SNHG5 knockdown. Further investigations showed that SNHG5 regulated the proliferation and CSC-like properties of HCC by binding UPF1 and regulating Wnt/ $\beta$-catenin pathway.

Conclusions: SNHG5 plays critical role to promote HCC cells proliferation and cancer stem cell-like properties via UPF1 and Wnt/ $\beta$-catenin pathway.

\section{Introduction}

Hepatocellular carcinoma (HCC) is the sixth prevalent human malignancy in the world, which is a kind of aggressive cancer and the primary histological type of liver malignancies ${ }^{1,2}$. Despite the prevention, diagnosis and treatment of HCC have obtained greatly development, the rates of recurrence and metastasis are still high, and the patients with HCC have a poor long survival ${ }^{3,4}$. Thus, it is significantly important and urgent to find out the mechanism of HCC progression, metastasis and recurrence, and investigate the new therapy method.

Heterogeneity is a characteristic and hallmark of tumor cells, which may be one of causes of tumor growth, metastasis and recurrence. It has been revealed that heterogeneity may result from groups of stem-like cells, which are regarded as the cancer stem cells (CSCs) ${ }^{5}$. CSCs have the abilities to self-renew, differentiate, keep uncontrollable growth, give rise to a new tumor in local or distant organs and have the interconversion with non-CSCs ${ }^{6,7}$. These features may mainly contribute to the progression, metastasis and recurrence of cancers ${ }^{8}$. The existence of liver CSCs has been verified. This group of cells has been distinguished, and they are characterized by several makers, such as CD133, CD13, CD90, and EpCAM 9-12. It has been revealed that several biological mechanisms are responsible to the functions of hepatic CSCs, such as AKT, transforming growth factor-beta (TGF- $\beta$ ) and so on.

Long noncoding RNAs (IncRNAs) called a kind of "dark matter" in human diseases are longer than 200nt, which could not code proteins ${ }^{13}$. More and more researches have revealed that IncRNAs could bind to DNA and RNA by a complementary sequence, playing critical roles in gene, such as transcription, mRNA 
splicing, RNA decay and translation ${ }^{14}$. And the posttranslational modification of proteins can be modulated by IncRNAs. LncRNAs play important roles in the proliferation, apoptosis and metastasis of cancers, which involved in different approaches, such as epigenetic silencing, splicing regulation, IncRNAmiRNA interaction, IncRNA-protein interaction and genetic variation, including $\mathrm{HCC}^{15}$. LncRNAs are contribute to liver cancer pathology, progression, outcomes and the maintenance of biological properties of liver CSCs, such as LncTCF7, IncBRM and IncCAMTA1 16-19.

It has been found that IncSNHG5 takes part in regulating different cancers. In this study, we aim to investigate the effect of IncSNHG5 on HCC and liver cancer stem cell-like properties.

\section{Materials And Methods}

\section{Cell lines and cell culture}

The human HCC cell line HepG2 and Huh7 were obtained from the Chinese Academy of Sciences Cell Bank (Shanghai, China). The cells were cultured in the DMEM/ High Glucose (hyclone, USA) in a humidified incubator at $37^{\circ} \mathrm{C}$ temperature and $5 \% \mathrm{CO}_{2}$ concentration. $10 \%$ FBS (Fetal bovine serum, Gibco USA) and Penicillin-Streptomycin $(100 \mathrm{U} / \mathrm{mL}$ and $100 \mu \mathrm{g} / \mathrm{mL}$ respectively) supplementations were added in the DMEM/ High Glucose medium prior to culture.

\section{Constructions of Plasmid and cell transfection}

SNHG5 and UPF1 overexpression plasmids, SNHG5 knockdown plasmids (SNHG5 shRNA with a corresponding negative control shRNA-NC), SNHG5-Mut/WT plasmids (pCMV-SNHG5- Mut vector containing mutations at the putative UPF1 binding site were generated by site-directed mutagenesis) and the siRNA (small interfering RNA) against UPF1, were designed by Genepharma ((Shanghai, China). Lipofectamine 2000 (Invitrogen, Carlsbad, CA, USA) was used to transfect the HCC cells with plasmids by the manufacture's protocol. The stable clones were selected by $5 \mu \mathrm{g} / \mathrm{ml}$ Puromycin contain medium. The Puromycin-resistant cell clones were established after 4 weeks. Gene expression level was evaluated by Quantitative real-time PCR.

\section{Cell proliferation assays}

MTT (3-[4, 5-dimethylthiazol-2-yl]-2,5-diphenyltetrazolium bromide) assay MTT(0.5 mg/ml) added into transfected cells and keeping in the dark for 4 hours. Then, removed the supernatant, added $150 \mu$ DMSO and measured the optical density (OD) at $490 \mathrm{~nm}$.

EdU incorporation assay Transfected cells were seeded in to a 96 well plate $\left(2 \times 10^{3}\right)$ with complete growth meium. Then according to the manufactures protocol with the EdU detection kits (Keygen, Nanjing, China) the process was carried out. The experiments were done in triplets. 
Colony formation assay After routine incubation, transfected cells were trypsinized, centrifuged, counted and replated at a density of 500 cells $/ 6 \mathrm{~cm}$ plate. After 12 days, the cell colonies (one colony containing at least 50 cells) were fixed with $3.7 \%$ methanol, stained with $0.1 \%$ crystal violet and counted.

\section{Sphere-formation assays}

Ultra-low attachment culture dishes (Corning, USA) were used to culture HepG2 and Huh7 cells with DMEM/F12 (Gibco, USA) added with 1\% FBS, $20 \mathrm{ng} / \mathrm{mL}$ epithelial growth factor, and $20 \mathrm{ng} / \mathrm{mL}$ fibroblast growth factor for two weeks. The formation and the number of spheroids were detected by a stereomicroscope (Olympus, Japan).

\section{RNA isolation and quantitative real-time PCR}

The total RNA from the cultured cells and collected HCC tissues were extracted form Trizol reagent (Invitrogen, Carlsbad, CA, USA) according to manufacture's protocol. The Prime Script TM RT Master Mix Kit (Takara, Japan) and Mir-X miRNA qRT-PCR SYBR Kit (Takara, Japan) were used to obtain the cDNA. Quantitative real-time PCR (qRT-PCR) was performed with SYBR Premix Ex Taq ${ }^{\text {TM }}$ II (Takara)on Thermal Cycler CFX6 System (Bio- Rad). $\beta$-actin as the endogenous controls of qRT-PCR. The $2^{-\triangle \Delta C t}$ method was used o calculate relative gene expression. Primers sequences for PCR were presented in Supplementary Table1.

\section{Western blot analysis}

The total protein from the cultured HCC cells and the tissue samples were isolated by RIPA (Beyotime, Haimen China) supplemented with proteinase and phosphatase. BCA detection kit (Keygen, Nanjing, China) was used for qualification according to the manufacture's protocol. For electrophoresis $5 \% \mathrm{gel}$ was used for concentration and $10 \%$ for separation. Following electrophoresis the proteins were transferred on a PVDF membrane (Merck Milipore) and were blocked by $5 \%$ non-fat milk for 1 hour. Then the PVDF membrane was incubated overnight at $4^{0} \mathrm{C}$ with the primary antibodies (Supplementary Table 2). On the next day the secondary antibody (Zhuangzhi Biology, China) was diluted 1:5000 ratio in TBST, and the membrane was re-incubated for 1 hour. The protein bands were evaluated by ECL immunoblotting kit following the manufacture's protocol (Milipore, USA).

\section{Immunofluorescence (IF)}

Cells were fixed in $4 \%$ paraformaldehyde at room temperature for 15 min after culturing on glass coverslips for $24 \mathrm{~h}$. Then washed by PBS. The adherent cells were permeabilized using $0.5 \%$ Triton X-100, blocked with $10 \%$ goat serum in for $1 \mathrm{~h}$. Then, incubated with primary antibody $4{ }^{\circ} \mathrm{C}$ overnight and secondary antibodies with an appropriate dilution. After washing three times with PBS, coverslips were stained with DAPI and imaged with a invert fluorescent microscope (Nikon Eclipse Ti-S).

\section{Tumor formation in BALB/c nude mice}


4 weeks aged BALB/c nude mice were randomly divided into two groups, which were purchased from the Central Laboratory of Animal Science, Xi'an Jiaotong University, China. The mice were kept under Sterile Specific-Pathogen free (SPF) environment. Then, $5 \times 10^{6} / 200 \mu l$ HepG2 cells stably transfected with SNHG5-shRNA or NC-shRNA were subcutaneously injected. The tumor formations were observed every 4 days interval. After 5 weeks injecting, mice were sacrificed. The study was done according to the Guide line for the "Care and Use of Laboratory Animals of the National Institutes of Health" and was approved by the Medical Ethics Committee of the Experimental Animal Center of Xi'an Jiaotong University.

\section{Luciferase reporter assay}

Before transfection the HCC cells were placed and cultured in a 96 well plate til $60-80 \%$ proliferation was achieved. Wild and mutant reporter plasmids of SNHG5 (SNHG5-WT-luc, SNHG5-MuT-luc) were cotransfected with UPF1 plasmid respectively. The alteration in the luciferase activity was devaluated in each group by Dual Luciferase Assay Kit and as a control Renilla luciferase activity was employed.

\section{Immunoprecipitation (RIP)}

Millipore EZ-Magna RIP RNA Binding Protein Immunoprecipitation kit (Millipore) was applied to performed RIP assays according to the manufacturer's protocol. Rabbit polyclonal IgG (Millipore) and antibodies to UPF1 (Abcam) were applied in RIP assays. Then, RIP-PCR was performed, and total RNA was used as input controls.

\section{Statistical analysis}

SPSS 23.0 (IBM, SPSS, Chicago, IL, USA) and GraphPad Prism V7.0 (GraphPad Software, CA, USA) were used to analyzed the results. Student's t-test was done to evaluate the difference between two groups. $A$ two-tailed $P<0.05$ was considered as statistically significant and $P<0.01$ was very significant.

\section{Results}

\section{Upregulation of SNHG5 promotes HCC cells proliferation in vitro}

The effect of SNHG5 on HCC cells in vitro was investigated by upregulating the expression of SNHG5. In order to perform this, we conducted the PCMV-SNHG5 expression plasmid, and transfected it into HepG2 and Huh7 cells. As a result, comparing with the empty vector, the expression of SNHG5 dramatically increased after transfection with the pCMV-SNHG5 vector in HepG2 and Huh7 cells (Figure 1A). Then growth curve of MTT assay showed that overexpression of SNHG5 could promote HepG2 and Huh7 cells proliferation (Figure 1B). Next, both the result of Edu assay and plate colony formation assays revealed the same growth trend of HCC cells with upregulation of SNHG5 (Figure 1C, D).

\section{Downregulation of SNHG5 represses HCC cells proliferation in vitro}


To further evaluate the role of SNHG5 on HCC cells proliferation, lentivirus-mediated short hairpins RNA (shRNA) was used to silence the SNHG5 (SNHG5-shRNA). The result of qRT-PCR showed that the expression of SNHG5 reduced highly in HepG2 and Huh 7 (Figure 2A). MTT, Edu assay and plate colony formation assays showed that knockdown of SNHG5 impaired the HepG2 and Huh 7 proliferation (Figure $2 B, C, D)$. These results suggest that SNHG5 is critical to HCC cells proliferation.

\section{Knockdown of SNHG5 represses tumor growth in vivo}

HepG2 and Huh 7 cells stably transfected with SNHG5-shRNA and NC were subcutaneously injected in male nude mice for 5 weeks. Compared with NC group, HCC cells transfected with SNHG5-shRNA greatly inhibited tumor growth (Figure $3 \mathrm{~A}$ ). We also observed the tumor volume and tumor weight in two group. And the tumor volume curve and tumor weight indicated that downregulation of SNHG5 suppressed tumor growth effectively (Figure 3B, C). Additionally, the expression of SNHG5 was detected in xenograft tumors. The result of qRT-PCR indicated that the expression of SNHG5 greatly decreased in SNHG5shRNA xenograft tumors (Figure 3D).

\section{Knockdown of SNHG5 represses the liver CSC-like properties}

To analyze the role of SNHG5 in liver CSC self-renewal, SNHG5-shRNA was used to deplete the expression of SNHG5 in HepG2 and Huh7 cells. And qRT-PCR was performed to detect the expression of SNHG5 in liver CSCs, which showed that the SNHG5 expression decrease in liver CSCs (Figure 4A). Then, the sphereformation assays were performed in the cells with SNHG5-shRNA and sh-NC, we found that the sphere formation was dramatically impared in SNHG5-shRNA cells compared with the sh-NC cells (Figure 4B, C). We also detected the makers of stem cells and the transcription factors in two CSCs groups. The result of qRT-PCR showed that the expression of CD133, CD44, ALDH1, Oct4, Sox2 and Nanog decreased in CSCs derived from SNHG5-shRNA cells (Figure 4D). The IF and Western Blot analysis verified the result of qtPCR (Figure 4E, F). These results indicated that IncSNHG5 was required in liver CSC-like properties.

\section{Depletion of SNHG5 increases the expression of UPF1}

It has been revealed that RNA-binding proteins (RBPs) play critical roles in IncRNA related pathway. And interacting with RNA-binding proteins (RBPs) is one of approached for IncRNAs involved in regulations. In order to investigate this, biological information prediction was performed including StarBase and DIANA LncBase software to find the proteins associated with SNHG5. Then, we noticed that there was a potential binding site for UPF1.

We found that the expression of UPF1 increased in HepG2 and Huh7 cells with depletion of SNHG5 (Figure 5A). Then, qRT-PCR and Western Blot were performed to analysis the expression of UPF1in liver CSCs, revealing that knockdown of SNHG5 could upregulate the expression of UPF1 (Figure 5B, C). Consistently, IF verified that UPF1 highly expressed in sh-SNHG5 liver CSCs (Figure 5D). To further investigate the interaction between SNHG5 and UPF1, luciferase reporter vectors of SNHG5 were constructed. The result of luciferase reporter assay showed that luciferase reporter activity was greatly 
promoted by co- transfecting cells with pCMV-SNHG5-WT and UPF1 vector, while co-transfecting cells with pCMV-SNHG5-Mut and UPF1 vector inhibited luciferase reporter activity (Figure 5E). Then, RNA immunoprecipitation (RIP) was performed to deeply detected the interaction between SNHG5 and UPF1. An antibody extracts from HCC cell lines HepG2 and HuH7, which was against UPF1. We found that SNHG5 enriched with UPF1 antibody, comparing to the non -specific antibody (IgG control; Figure 5F). These results suggested that SNHG5 interacts with UPF1, and the expression of SNHG5 and UPF1 was opposite tendency.

\section{Downregulation of UPF1 increases liver CSC-like properties}

To explore the role of UPF1 in the sphere-formation of liver CSCs, we depleted the expression of UPF1 by introducing two specific siRNAs (siRNA \#1, \#2) against UPF1 gene transcript into HepG2 and Huh7 cells. We performed sphere-formation assays, finding that the liver CSC-like properties were promoted in HCC cells with si-UPF1 (Figure 6A). And the number of spheres per 1000 single liver CSCs increased after UPF1 knockdown (Figure 6B). Then, qRT-PCR was performed to detected the expression of markers (ALDH1, CD44, and CD133) and stem factors (NANOG, OCT4, and SOX2), which were upregulated with depletion of UPF1 (Figure 6C). Meanwhile, this result was verified in Western Blot analysis (Figure 6D). These results suggested that UPF1 played a critical role in liver CSCs formation.

\section{Wnt/ $\beta$-catenin pathway is responsible for liver CSC-like properties}

It has been proved that Wnt/ $\beta$-catenin pathway played a critical role in HCC, and SNHG5 took part in activation of $\mathrm{Wnt} / \beta$-catenin pathway. Hence, we speculated that $\mathrm{Wnt} / \beta$-catenin pathway may be a key factor to liver CSC-like properties. To investigate this, we extracted proteins from HepG2 CSCs and Huh7 CSCs and performed Western Blot analysis. The result showed that the expressions of Wnt1, Wnt3a and Wnt10a were downregulated with depletion of SNHG5, while they were upregulated with knockdown of UPF1 (Figure 7A). The result was confirmed in qRT-PCR (Figure 7B). $\beta$-catenin is one of the key factors in Wnt/ $\beta$-catenin pathway, and we detected the expression of $\beta$-catenin in HepG2 CSCs and Huh7 CSCs with IF analysis. We observed that the expression of $\beta$-catenin increased in liver CSCs with depletion of UPF1, while $\beta$-catenin decreased in liver CSCs with downregulation of SNHG5 (Figure 7C), as same result shown in qRT-PCR (Figure 7D). Consistently, critical components of Wnt/ $\beta$-catenin pathway ( $\beta$-catenin, TCF4, cmyc, cyclinD1, c-Jun) were detected in HepG2 CSCs and Huh7 CSCs on protein level via Western Blot analysis. We observed that these key components were downregulated with knockdown SNHG5, while their expression increased with depletion of UPF1 (Figure 7E). To further prove this, XAV-939,a Wnt/ $\beta$ catenin pathway inhibitor was adopted. With XAV-939, the sphere-formation of liver CSCs was impared and the number of spheres per 1000 single liver CSCs decreased (Figure 7F, G). These results suggested that SNHG5 could activated Wnt/ $\beta$-catenin pathway, and the activation of Wnt/ $\beta$-catenin pathway could promote the liver CSC-like properties.

\section{Discussion}


LncSNHG5 has been investigated in many solid cancers, such as colorectal cancer, gastric cancer and osteosarcoma, even myeloid leukemia ${ }^{20-23}$. And the mechanisms were detected. LncSNHG5 could promote the growth and(or) metastasis of osteosarcoma cells by suppressing the miR-212-3p/SGK3 signaling pathway, and it also plays a role of competing endogenous RNAs (ceRNAs) to regulate gastric cancer through miR-32 targeting KLF4, and contribute to the resistance of imatinib in chronic myeloid leukemia through miR-205-5p 22,23. In our recent study, we found that knockdown of IncSNHG5 could contribute to the growth of HCC cells, while the upregulation showed the opposite tendency.

CSCs is a kind of cells with characters of self-renew and differentiation, which has been observed and isolated in many solid cancers, such as colorectal cancer, lung cancer, and breast cancer ${ }^{24-26}$. Liver CSCs also was identified, which contained various subtypes characterized by different surface makers, such as $\mathrm{CD} 133^{+} \mathrm{CD} 13^{+}$, $\mathrm{EpCAM}^{+} \mathrm{CD} 24^{+} \mathrm{OV}^{+}$and $\mathrm{CD} 133^{+} \mathrm{CD} 44^{+} \mathrm{CD} 24^{+} \mathrm{EpCAM}^{+}$and so on ${ }^{27}$.

CSCs contribute to the epithelial-mesenchymal transition (EMT), metastasis, drug resistance and radio resistance though varieties of mechanisms. ${ }^{26,28-30}$ One of the important conception involved in properties of CSCs is cancer microenvironment. And T cells and tumor-associated macrophages (TAMS) take part in this complex and interacted network as well ${ }^{26}$. Recently, it has been revealed that IncRNAs played critical roles in CSCs. In breast cancer, IncRNA HOTAIR contributes to the EMT through HoxD10/miRNA 7/SETDB1/STAT3 pathway ${ }^{26}$. Besides the tumor promoting effects of IncRNAs on CSCs, the inhibitory role of IncRNAs also exists. In glioma, the downregulation of IncRNA-ROR could promote the proliferation of cancer cells and the formation of sphere of stem cells with the down expression of stem cell factor KLF4 ${ }^{27}$. In HCC, it has been verified that many IncRNAs are responsible to drive CSCs selfrenewal and tumor progression though various mechanisms. For example, IncSox 4 increased in liver CSCs could promote the self-renew of CSCs and tumorigenic capacity through STAT3 pathway ${ }^{31}$. LncTCF7 also was increased in liver CSCs and contribute to the maintenance of self-renew and tumorigenic capacity of $\mathrm{CSCs}^{17}$. Nevertheless, recent study has revealed that IncDILC was downregulated in liver CSCs and had the suppressive function of spheroid-formed, promoting the expansion of liver CSCs via IL6/STAT3 pathway ${ }^{32}$. In this research, we investigated and analyzed the function of IncSNHG5 in HCC cells and liver CSCs. The results suggested that depletion of IncSNHG5 suppressed the growth of cancer cells and stem cell-like properties. The surface makers and several stem factors were upregulated with the increasement of IncSNHG5 expression.

Mechanistically, we found that the IncSNHG5 combined with UPF1, and the overexpression of IncSNHG5 following the downregulation of UPF1 with the down-expression of surface makers and stem factors.

Recently, several researches demonstrated that Wnt/ $\beta$-Catenin signaling played a pivotal role in cancer stem cells. For example, in glioma, the self-renewal and tumorigenicity of CSCs were regulated by dysregulated Wnt-FoxM1/ $\beta$-Catenin signaling pathway ${ }^{33}$. In our study, the upregulation of IncSNHG5 could activate the Wnt/ $\beta$-Catenin pathway, and several expressions of critical components of the pathway were increased. And the formation of CSCs spheres was promoted. To detect the mechanisms 
further, the inhibitory of Wnt/ $\beta$-Catenin pathway, XAV-939 was used. The results showed the stem cell-like properties were inhibited.

Our study investigated the important function of IncSNHG5 in HCC cell lines and cancer stem cell-like properties, detecting the mechanism. However, the experiments for evaluating tumorigenicity of liver stem cells were not performed, and the effect of IncSNHG5 in liver CSCs on tumorigenicity was not evaluate either. Many researches have demonstrated that CSCs took part in a considerably complex network, tumor microenvironment(TME), which supported the malignancy of cancer cells and $\operatorname{CSCs}^{34}$.

Mesenchymal cells, tumor-associated macrophages and myeloid-derived suppressor cells (MDSCs) are usually involved in TME, which are associated with CSCs via various mechanisms ${ }^{35-37}$. In the further study, we aim to investigate the interaction between liver CSCs and the TME whether involving the IncSNHG5.

\section{Conclusions}

In summary, our research found that IncSNHG5 was related to the HCC cells proliferation in vitro and the tumor growth in vivo. The first time, we observed that IncSNHG5 was responsible to the sphere formation of liver CSCs and the HCC cells stem-like properties, and downregulation of UPF1 could increase liver CSC-like properties. In mechanism, the importance of Wnt/ß-Catenin pathway was reconfirmed in CSCs, especially in HCC in our study. At the same time, the study demonstrated that IncSNHG5 could active the Wnt/ $\beta$-Catenin pathway, promoting the HCC cells stem-like properties.

\section{Declarations}

\section{Ethics approval and consent to participate}

The study were approved by the ethics committee of the First Affiliated Hospital of Xi'an Jiaotong University. The animal experiments were performed according to the National Institutes of Health animal use guidelines on the use of experimental animals.

\section{Author information}

Author notes Yarui Li and Junbi Hu contributed equally to this work.

\section{Authors' contributions}

HSX conceived and designed the project. LYR and HJB carried out most of the experiments. HJB and ZZY wrote the manuscript, RMD and LGF is responsible for the animal experiments , GD and LXL performed the statistical analysis, all authors read and approved the final manuscript.

\section{Consent for publication}

Not applicable. 
Availability of data and material

The data and material in this study are available.

\section{Funding}

This work was supported by the Social Development, Scientific and Technological Research Project of Shaanxi Province (No. 2017ZDXM-SF-065).

\section{Competing interests}

The authors declare that they have no competing interests.

\section{Abbreviations}

Long noncoding RNAs (IncRNAs) 『Hepatocellular carcinoma (HCC), cancer stem cells (CSCs)区 transforming growth factor-beta (TGF- $\beta$ ) $\llbracket$ siRNA (small interfering RNA), optical density (OD), Quantitative real-time PCR (qRT-PCR), Immunofluorescence (IF), Sterile Specific-Pathogen free (SPF), Immunoprecipitation (RIP)

\section{References}

1. Quaglia A. Hepatocellular carcinoma: a review of diagnostic challenges for the pathologist. Journal of hepatocellular carcinoma. 2018;5:99-108.

2. Laursen L. A preventable cancer. Nature. 2014;516(7529):S2-3.

3. Tang ZY, Ye SL, Liu YK, et al. A decade's studies on metastasis of hepatocellular carcinoma. Journal of cancer research and clinical oncology. 2004;130(4):187-196.

4. Forner A, Llovet JM, Bruix J. Hepatocellular carcinoma. Lancet (London, England). 2012;379(9822):1245-1255.

5. Shackleton M, Quintana E, Fearon ER, Morrison SJ. Heterogeneity in cancer: cancer stem cells versus clonal evolution. Cell. 2009;138(5):822-829.

6. Singh AK, Arya RK, Maheshwari S, et al. Tumor heterogeneity and cancer stem cell paradigm: updates in concept, controversies and clinical relevance. International journal of cancer. 2015;136(9):1991-2000.

7. Cai Z, Xu K, Li Y, Lv Y, Bao J, Qiao L. Long noncoding RNA in liver cancer stem cells. Discovery medicine. 2017;24(131):87-93.

8. Ji J, Wang XW. Clinical implications of cancer stem cell biology in hepatocellular carcinoma. Seminars in oncology. 2012;39(4):461-472.

9. Yin S, Li J, Hu C, et al. CD133 positive hepatocellular carcinoma cells possess high capacity for tumorigenicity. International journal of cancer. 2007;120(7):1444-1450. 
10. Haraguchi $\mathrm{N}$, Ishii $\mathrm{H}$, Mimori $\mathrm{K}$, et al. CD13 is a therapeutic target in human liver cancer stem cells. The Journal of clinical investigation. 2010;120(9):3326-3339.

11. Yang ZF, Ho DW, Ng MN, et al. Significance of CD90+ cancer stem cells in human liver cancer. Cancer cell. 2008;13(2):153-166.

12. Yamashita T, Ji J, Budhu A, et al. EpCAM-positive hepatocellular carcinoma cells are tumor-initiating cells with stem/progenitor cell features. Gastroenterology. 2009;136(3):1012-1024.

13. Martin L, Chang HY. Uncovering the role of genomic "dark matter" in human disease. The Journal of clinical investigation. 2012;122(5):1589-1595.

14. Lv H, Lv G, Han Q, Yang W, Wang H. Noncoding RNAs in liver cancer stem cells: The big impact of little things. Cancer letters. 2018;418:51-63.

15. Huang JL, Zheng L, Hu YW, Wang Q. Characteristics of long non-coding RNA and its relation to hepatocellular carcinoma. Carcinogenesis. 2014;35(3):507-514.

16. Huo X, Han S, Wu G, et al. Dysregulated long noncoding RNAs (IncRNAs) in hepatocellular carcinoma: implications for tumorigenesis, disease progression, and liver cancer stem cells. Molecular cancer. 2017;16(1):165.

17. Wang Y, He L, Du Y, et al. The long noncoding RNA IncTCF7 promotes self-renewal of human liver cancer stem cells through activation of Wnt signaling. Cell stem cell. 2015;16(4):413-425.

18. Ding LJ, Li Y, Wang SD, et al. Long Noncoding RNA IncCAMTA1 Promotes Proliferation and Cancer Stem Cell-Like Properties of Liver Cancer by Inhibiting CAMTA1. International journal of molecular sciences. 2016;17(10).

19. Zhu P, Wang Y, Wu J, et al. LncBRM initiates YAP1 signalling activation to drive self-renewal of liver cancer stem cells. Nature communications. 2016;7:13608.

20. Zhao L, Han T, Li Y, et al. The IncRNA SNHG5/miR-32 axis regulates gastric cancer cell proliferation and migration by targeting KLF4. FASEB journal : official publication of the Federation of American Societies for Experimental Biology. 2017;31(3):893-903.

21. Damas ND, Marcatti M, Come C, et al. SNHG5 promotes colorectal cancer cell survival by counteracting STAU1-mediated mRNA destabilization. Nature communications. 2016;7:13875.

22. He B, Bai Y, Kang W, Zhang X, Jiang X. LncRNA SNHG5 regulates imatinib resistance in chronic myeloid leukemia via acting as a CeRNA against MiR-205-5p. American journal of cancer research. 2017;7(8):1704-1713.

23. Ju C, Zhou R, Sun J, et al. LncRNA SNHG5 promotes the progression of osteosarcoma by sponging the miR-212-3p/SGK3 axis. Cancer cell international. 2018;18:141.

24. Merlos-Suarez A, Barriga FM, Jung P, et al. The intestinal stem cell signature identifies colorectal cancer stem cells and predicts disease relapse. Cell stem cell. 2011;8(5):511-524.

25. MacDonagh L, Gray SG, Breen E, et al. Lung cancer stem cells: The root of resistance. Cancer letters. 2016;372(2):147-156. 
26. Geng SQ, Alexandrou AT, Li JJ. Breast cancer stem cells: Multiple capacities in tumor metastasis. Cancer letters. 2014;349(1):1-7.

27. Feng S, Yao J, Chen Y, et al. Expression and Functional Role of Reprogramming-Related Long Noncoding RNA (lincRNA-ROR) in Glioma. Journal of molecular neuroscience : MN. 2015;56(3):623630 .

28. Liu X, Fan D. The epithelial-mesenchymal transition and cancer stem cells: functional and mechanistic links. Current pharmaceutical design. 2015;21(10):1279-1291.

29. Leon G, MacDonagh L, Finn SP, Cuffe S, Barr MP. Cancer stem cells in drug resistant lung cancer: Targeting cell surface markers and signaling pathways. Pharmacology \& therapeutics. 2016;158:7190.

30. Chang L, Graham P, Hao J, et al. Cancer stem cells and signaling pathways in radioresistance. Oncotarget. 2016;7(10):11002-11017.

31. Chen ZZ, Huang L, Wu YH, Zhai WJ, Zhu PP, Gao YF. LncSox4 promotes the self-renewal of liver tumour-initiating cells through Stat3-mediated Sox4 expression. Nature communications. 2016;7:12598.

32. Wang X, Sun W, Shen W, et al. Long non-coding RNA DILC regulates liver cancer stem cells via IL6/STAT3 axis. Journal of hepatology. 2016;64(6):1283-1294.

33. Gong A, Huang S. FoxM1 and Wnt/beta-catenin signaling in glioma stem cells. Cancer research. 2012;72(22):5658-5662.

34. Kise K, Kinugasa-Katayama Y, Takakura N. Tumor microenvironment for cancer stem cells. Advanced drug delivery reviews. 2016;99(Pt B):197-205.

35. Cui TX, Kryczek I, Zhao L, et al. Myeloid-derived suppressor cells enhance stemness of cancer cells by inducing microRNA101 and suppressing the corepressor CtBP2. Immunity. 2013;39(3):611-621.

36. Yang J, Liao D, Chen C, et al. Tumor-associated macrophages regulate murine breast cancer stem cells through a novel paracrine EGFR/Stat3/Sox-2 signaling pathway. Stem cells (Dayton, Ohio). 2013;31(2):248-258.

37. Kinugasa Y, Matsui T, Takakura N. CD44 expressed on cancer-associated fibroblasts is a functional molecule supporting the stemness and drug resistance of malignant cancer cells in the tumor microenvironment. Stem cells (Dayton, Ohio). 2014;32(1):145-156.

\section{Figures}




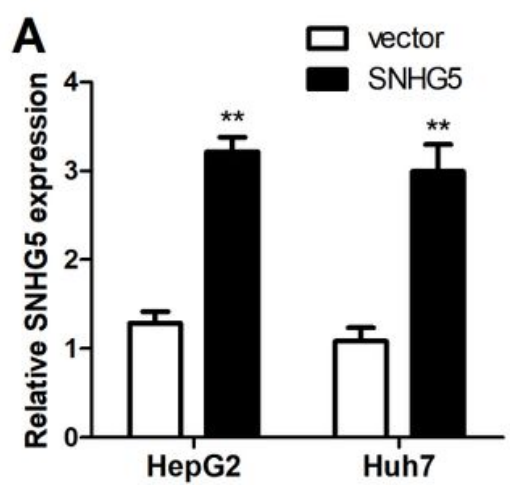

B
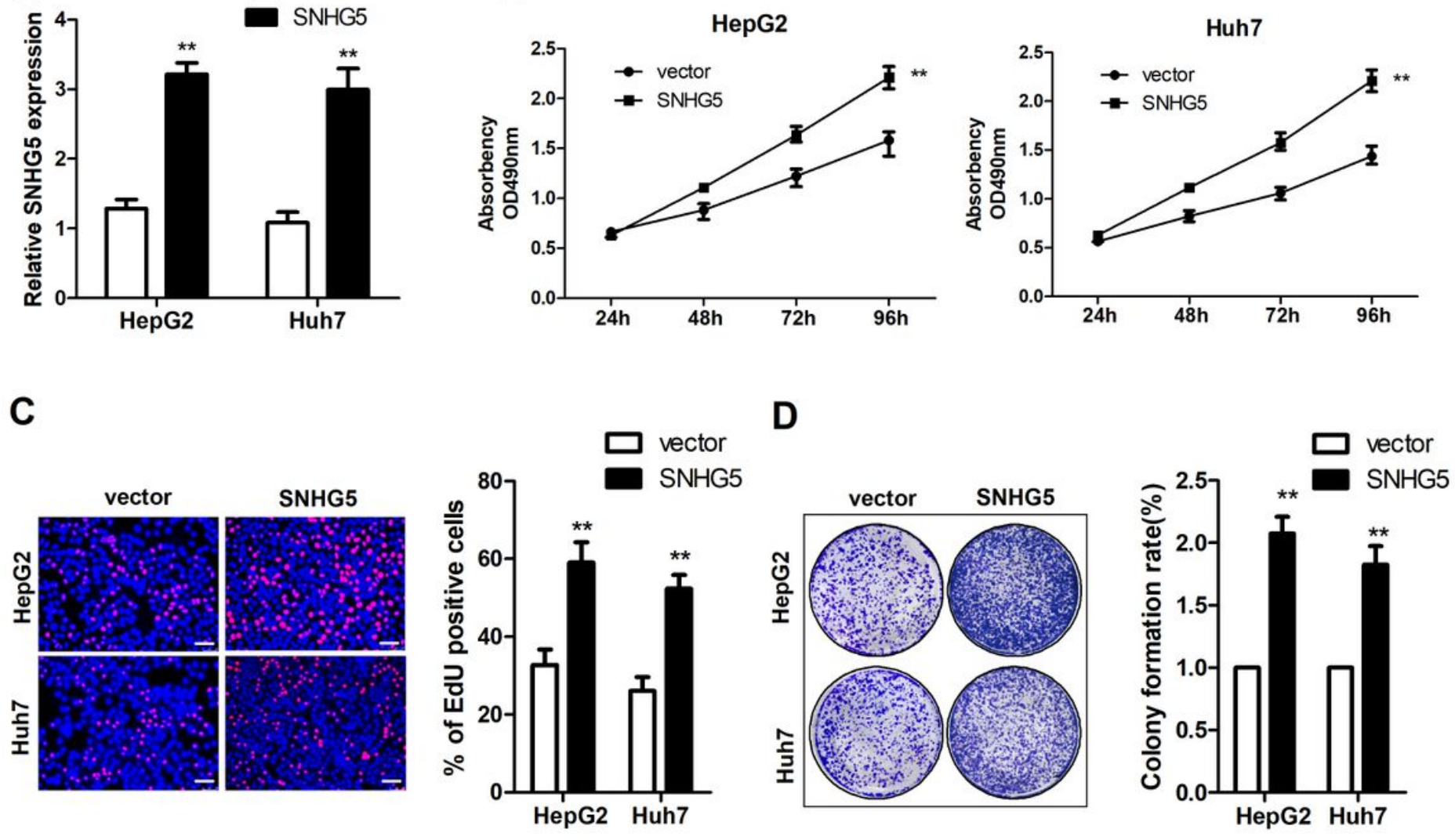

Figure 1

Overexpression of SNHG5 promotes HCC cell proliferation in vitro. (A) qRT-PCR analysis of SNHG5 expression after SNHG5 overexpression. MTT assays (B), EdU assay(C) and colony formation assay showed SNHG5 overexpression promotes HCC cells proliferation. ${ }^{\star \star} P<0.01$. White bar: $20 \mathrm{~mm}$. 
A

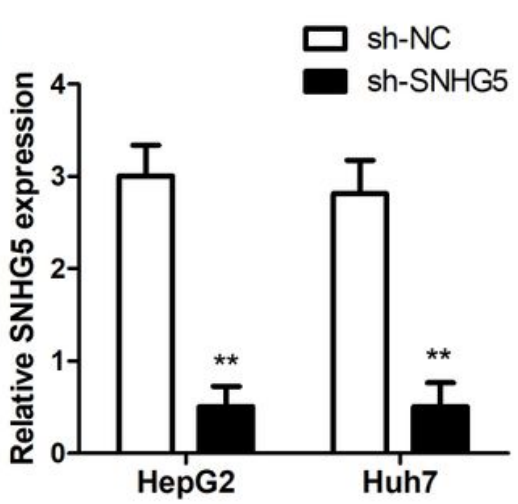

B
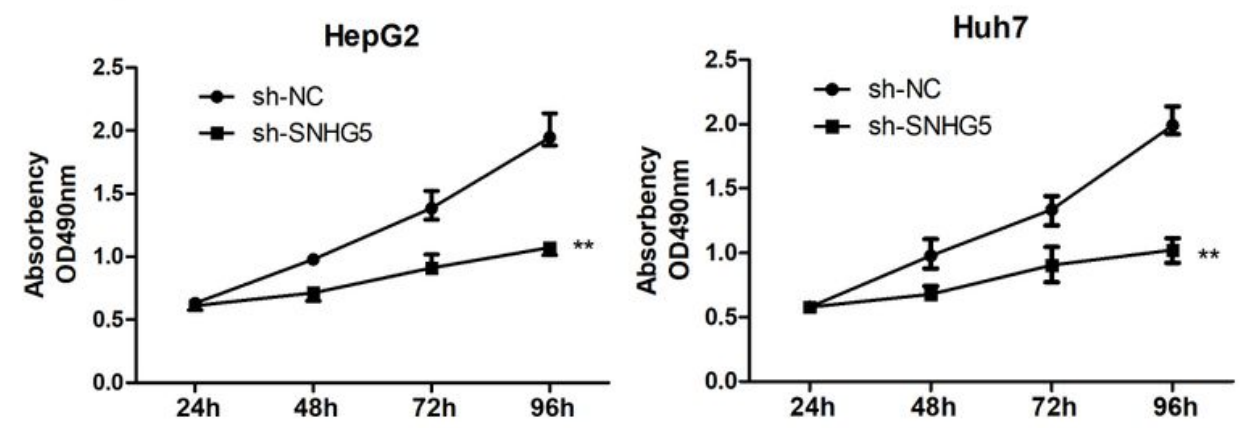

C

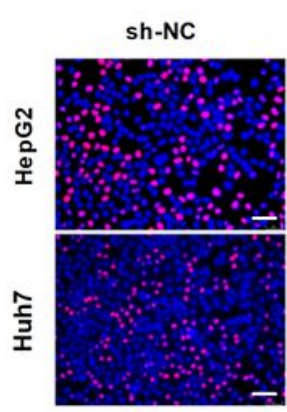

sh-SNHG5
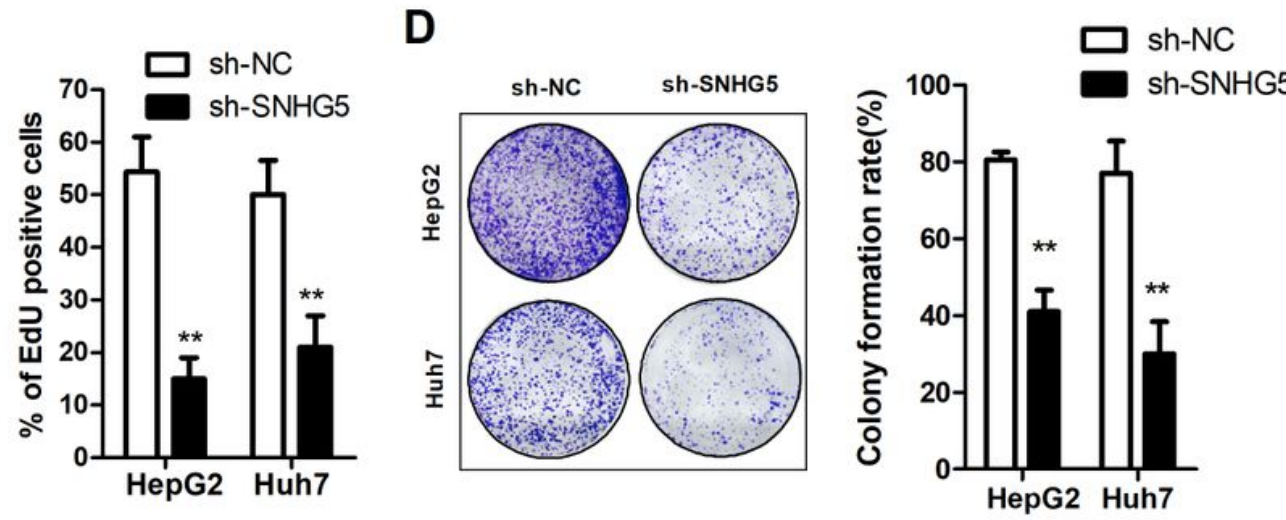

Figure 2

Konckdown of SNHG5 represses HCC cell proliferation in vitro. (A) qRT-PCR analysis of SNHG5 expression following transfected HCC cells with SNHG5-shRNA. MTT assays (B), EdU assay(C) and colony formation assay showed downregualtion of SNHG5 inhibits $\mathrm{HCC}$ cells proliferation. ${ }^{*} \mathrm{P}<0.01$. White bar: $20 \mathrm{~mm}$. 

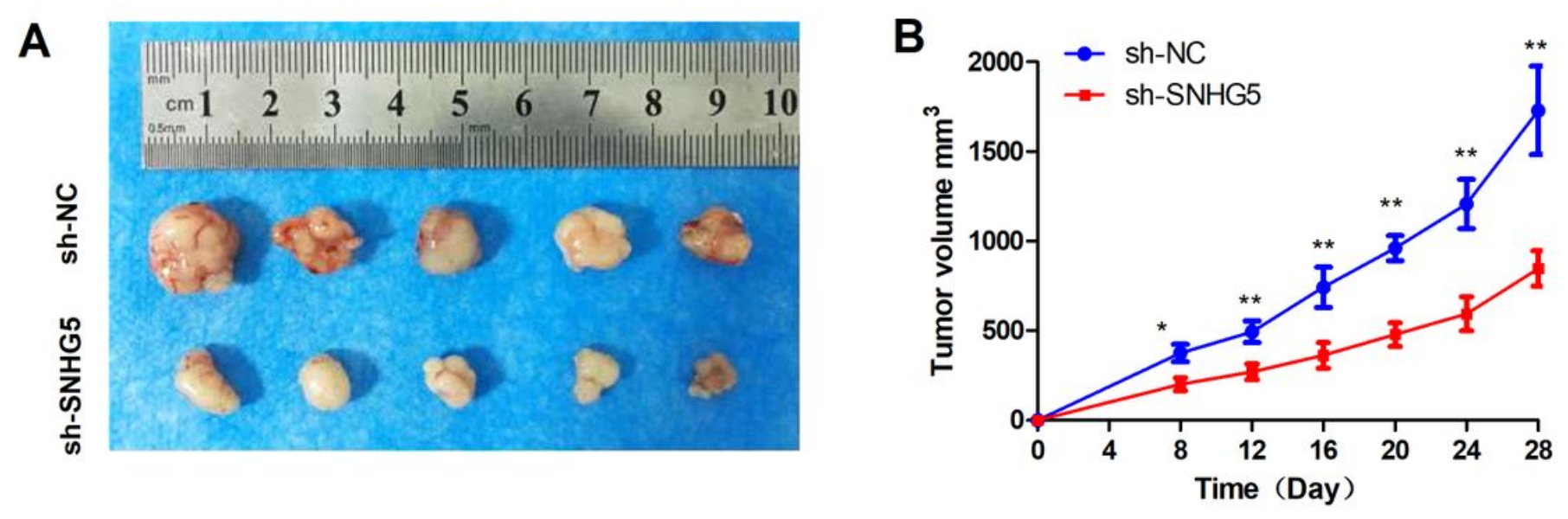

C

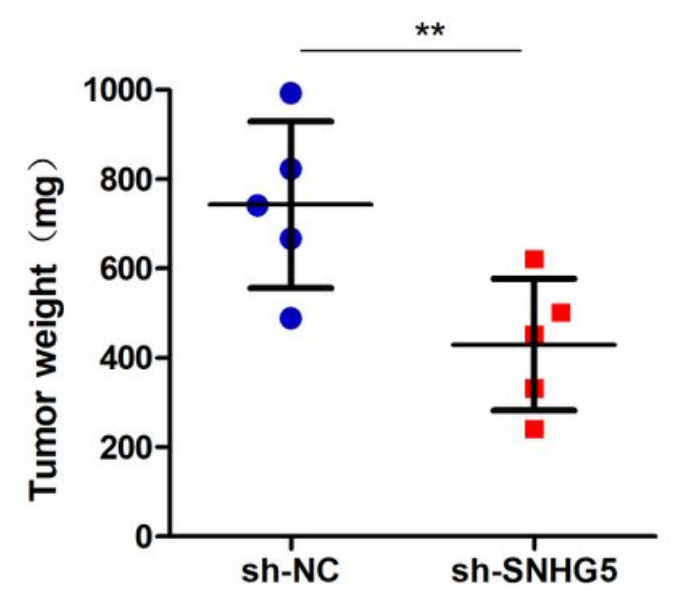

D

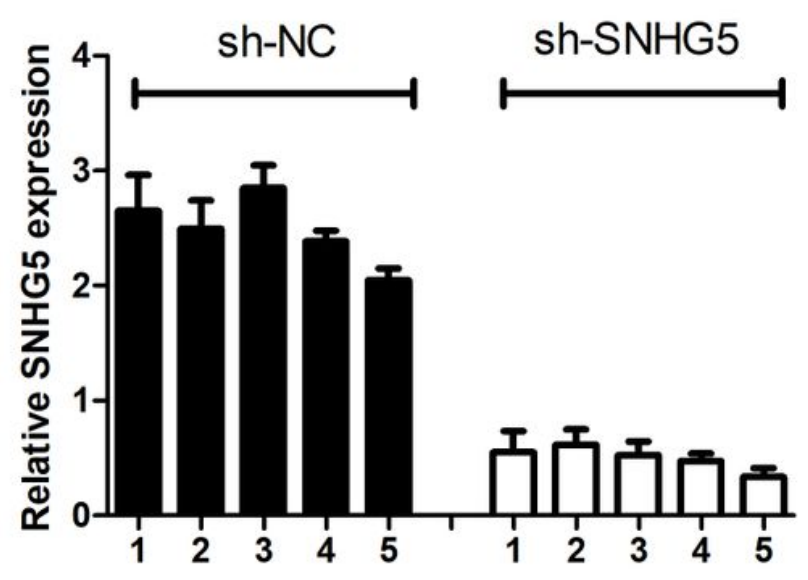

\section{Figure 3}

Knockdown of SNHG5 inhibits tumor growth in vivo. (A) Representative images of nude mice modles and formed tumors that were subcutaneously injected with SNHG5-shRNA and NC-shRNA cells. Effect of SNHG5 knockdown on HCC growth in vivo according to the tumor growth curve (B) and tumor weight(C) . ${ }^{*} P<0.05 ;{ }^{*} P<0.01$. (D) The expression of SNHG5 in xenograft tumors were detected by qRTPCR. ${ }^{* *} \mathrm{P}<0.01$. 
A
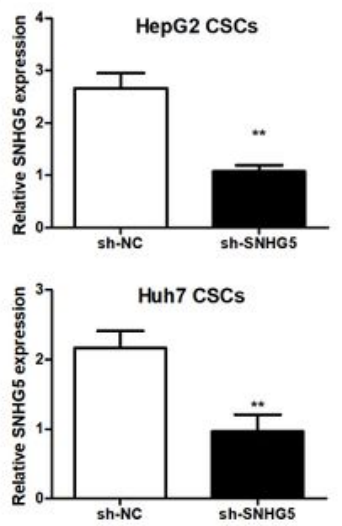

D

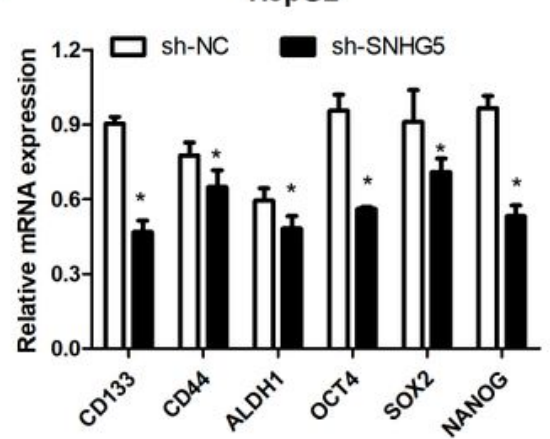

C
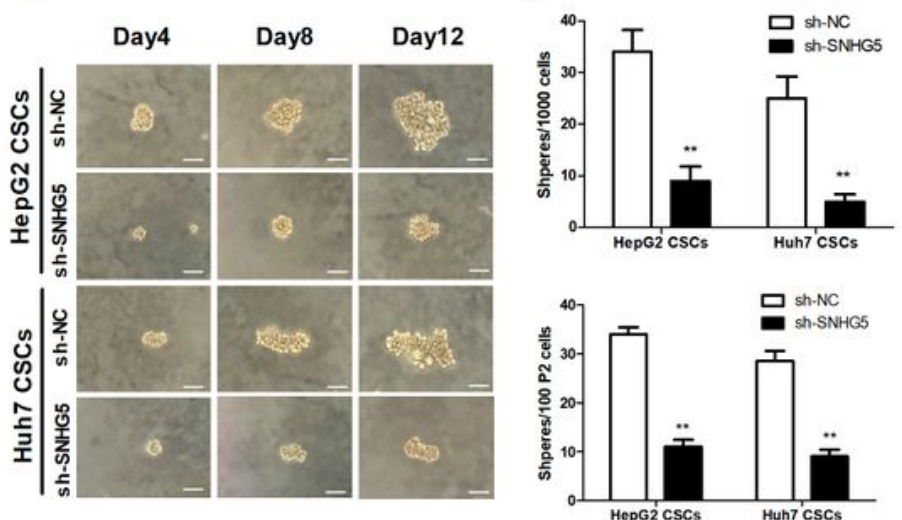

Huh7
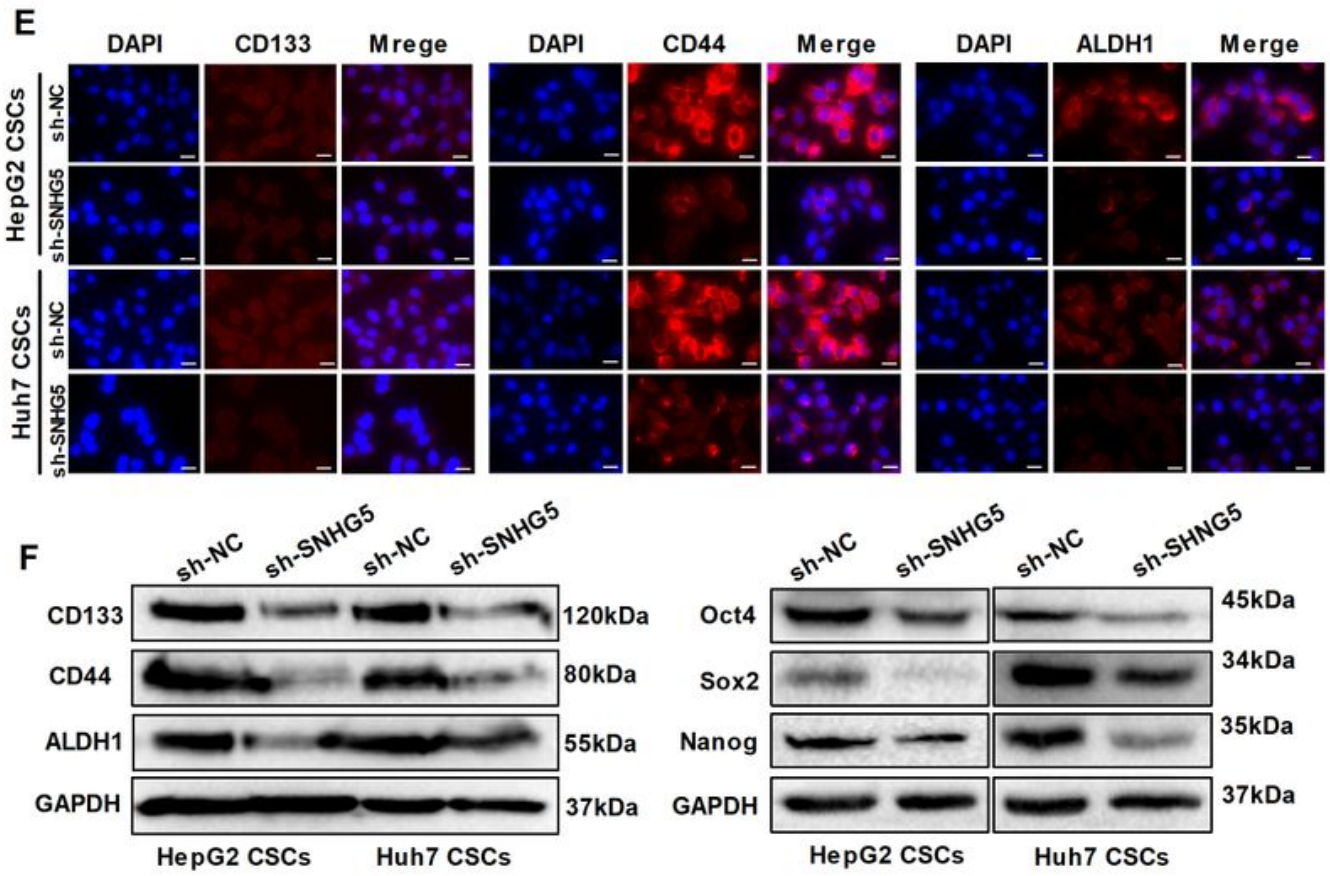

Figure 4

Knockdown of SNHG5 represses the liver CSC-like properties. (A) qRT-PCR of SNHG5 expression in HepG2 and Huh7 CSCs by sh-NC and sh-SNHG5. (B) Bright-field microscopy images showed the typical morphological features of small aggregates and spheres after knockdown of SNHG5 in HCC CSCs separately on the 4rd, 8th, or 12th day, white bar: $20 \mathrm{~mm}$. (C) Quantification of the total number of primary spheres per 1000 single CSCs and secondary spheres (P2) per 100 single CSCs after SNHG5 
knockdown. (D) qRT-PCR, (E) Immunofluorescence (IF) analysis and Western blotting analysis (F) of the expression of stem factors (SOX2, OCT4 and NANOG) and markers (CD133, CD44 and ALDH1) in shSNHG5 HepG2 and Huh7 CSCs compared with sh-NC cells. ${ }^{*} \mathrm{P}<0.05 ;{ }^{*} \mathrm{P}<0.01$. Results suggested that knocksown of SNHG5 inhibits CSC properties. White bar: $50 \mathrm{~mm}$.
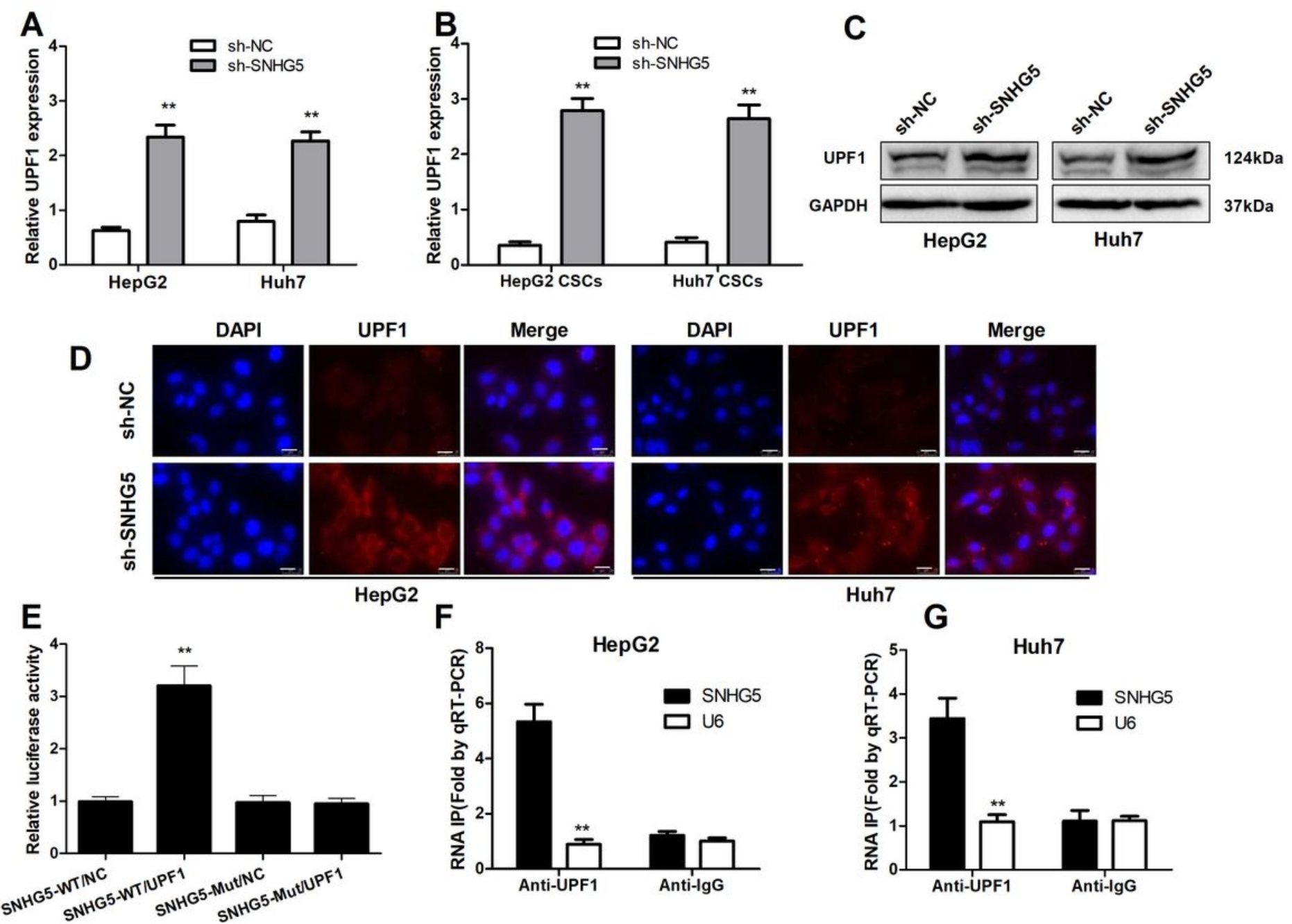

\section{Figure 5}

Depletion of SNHG5 increases the expression of UPF1. (A) qRT-PCR analysis of UPF1 expression following transfected HCC cells with SNHG5-shRNA. (B) qRT-PCR analysis of UPF1 expression in HCC CSCs. (C) UPF1 protein levels in HepG2 and Huh7 cells stably overexpressing when SNHG5 knockdown. (D) IF analysis the expression of UPF1 in sh-SNHG5 HepG2 and Huh7 cells compared with sh-NC cells. (E) Luciferase reporter assay showed that luciferase activity was greatly promoted by co-transfecting cells with pCMV-SNHG5-WT and UPF1 vector. (F,G) RNA immunoprecipitation (RIP) was performed to detected the interaction between SNHG5 and UPF1. ${ }^{*} P<0.05 ;{ }^{*} P<0.01$. White bar: $50 \mathrm{~mm}$. 
A

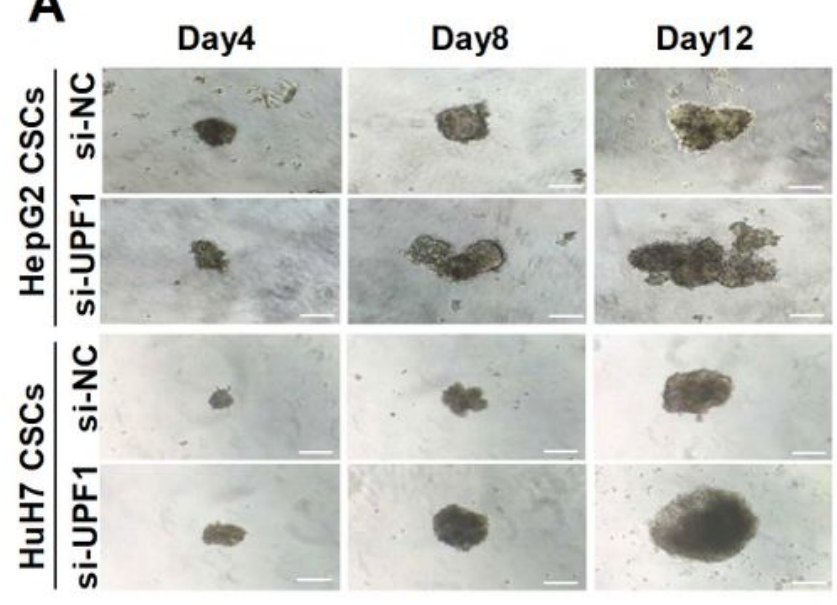

C

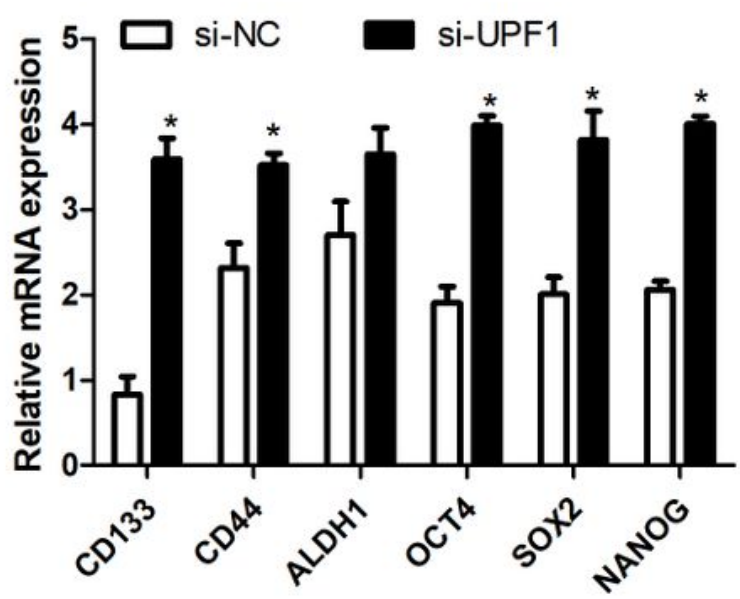

D

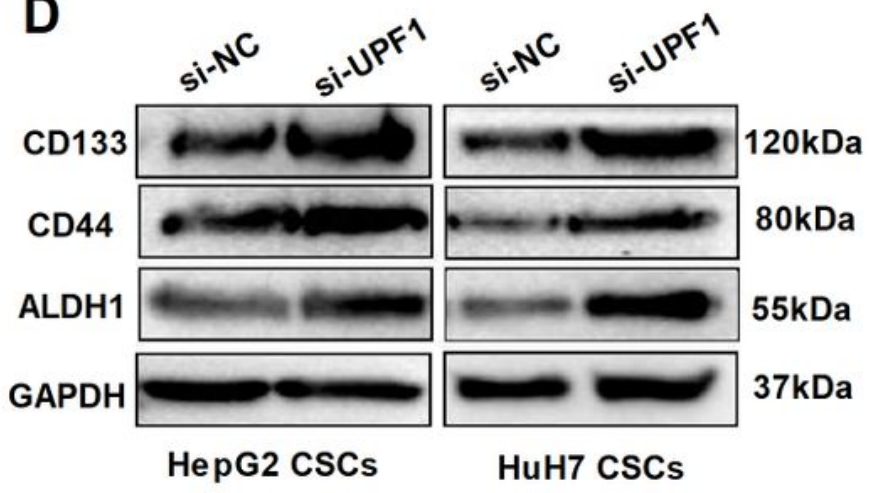

B

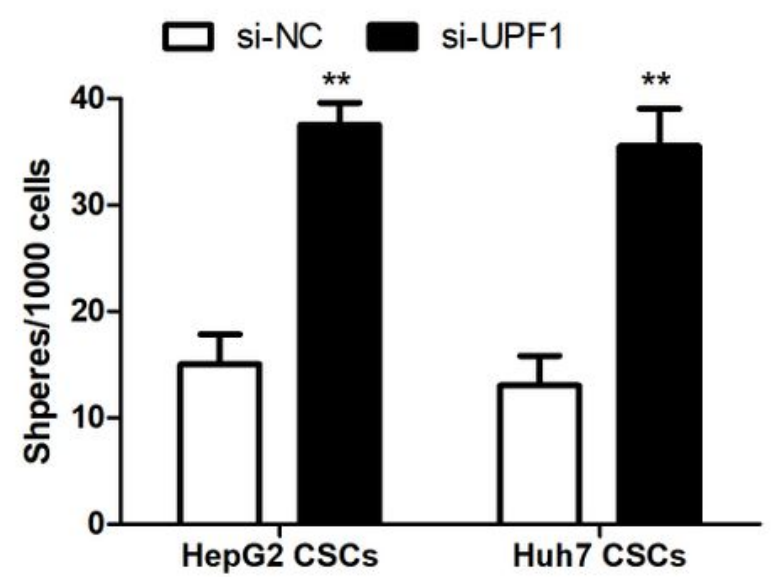

Huh7
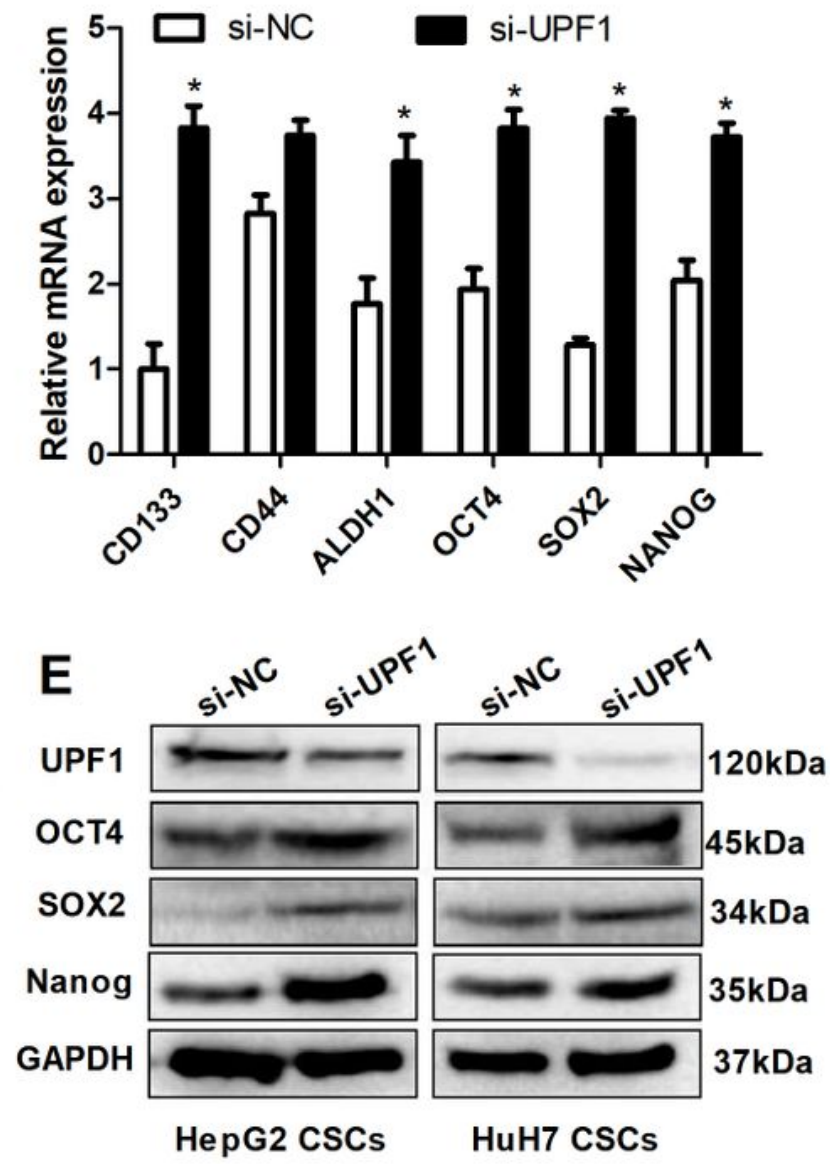

Figure 6

UPF1 is necessary for maintaining HCC CSC proliferation and self-renewal. (A) Bright-field microscopy images showed the typical morphological features of small aggregates and spheres after silencing of UPF1 in HCC CSCs separately on the 4rd, 8th, or 12th day, white bar: $50 \mathrm{~mm}$. White bar: $20 \mathrm{~mm}$.(B) Quantification of the total number of primary spheres per 1000 single CSCs after UPF1 knockdown. (C) qRT-PCR and (D) Western blotting analysis of the expression of stem factors (SOX2, OCT4 and NANOG) 
and markers (CD133, CD44 and ALDH1) in si-UPF1 HepG2 and Huh7 cells compared with si-NC cells. *P< $0.05 ; * * \mathrm{P}<0.01$.

A

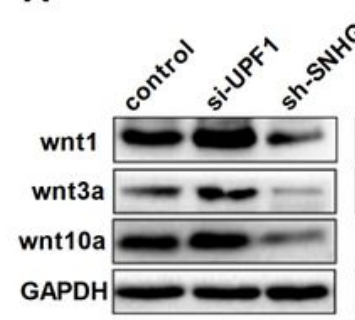

HepG2 CSCs

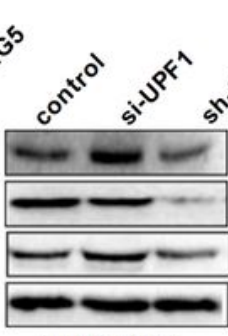

Huh7 CSCs
B
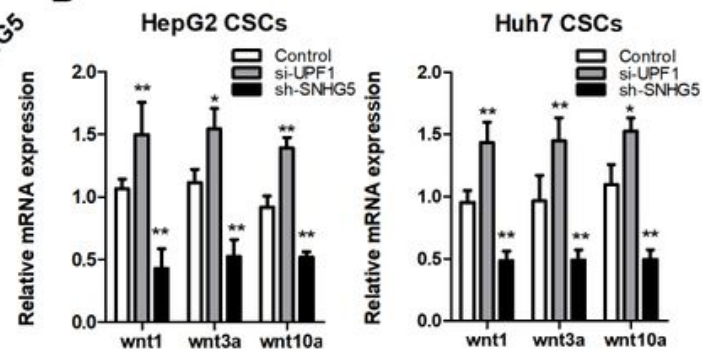

C
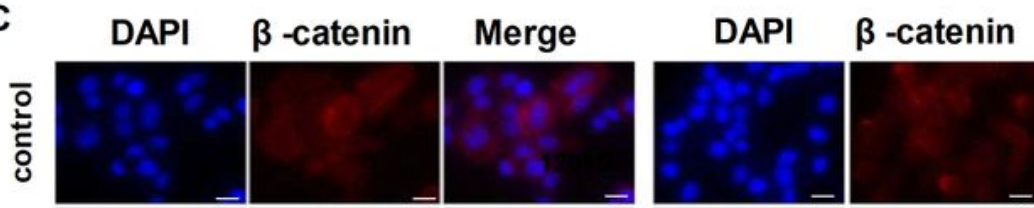

Merge

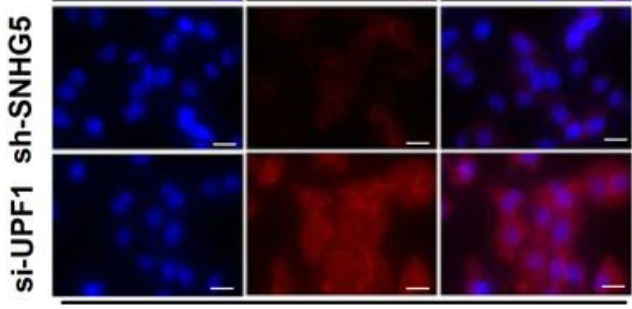

HepG2 CSCs

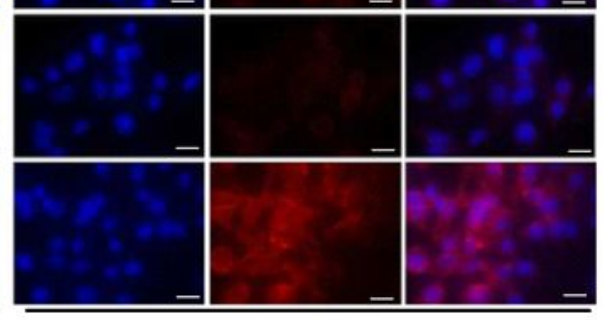

Huh7 CSCs

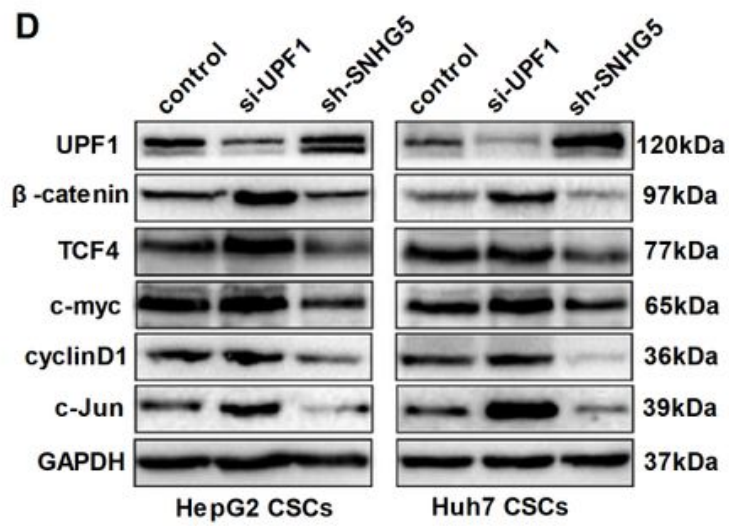

E

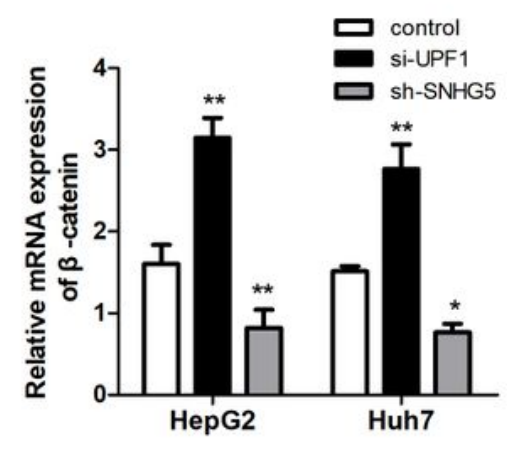

F
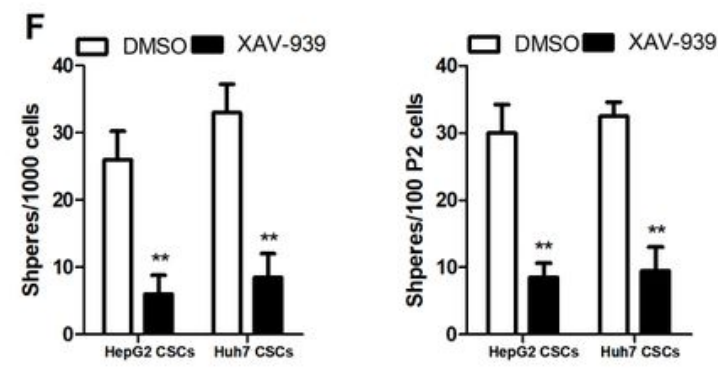

\section{Figure 7}

SNHG5/UPF1 axis enhanced CSC properties through the Wnt/ $\beta$-catenin pathway. (A) Western bloting analysis of the protein levels of Wnt family members (Wnt, Wnt3a, Wnt10a) in sh-SNHG5, si-UPF1 and Control cells.(B) qRT-PCR analysis of the mRNA levels of Wnt family members (Wnt, Wnt3a, Wnt10a) in 
sh-SNHG5, si-UPF1 and Control cells. (C) $\beta$-catenin immunofluorescence of seeded HCC CSCs. White bar: $50 \mathrm{~mm}$. (D) Western bloting analysis of the protein levels of UPF1 and the key factors of Wnt/ $\beta$-catenin pathway ( $\beta$-catenin, c-Jun, TCF-4, cyclinD1, c-myc). (E) qRT-PCR analysis of the mRNA levels of $\beta$-catenin in sh-SNHG5, si-UPF1 and Control cells. (F) Bright-field microscopy images showed the number of spheres derived from Wnt inhibitors XAV-939 at the 12th day of sphere formation culture. XAV-939 reduced sphere formation. White bar: $20 \mathrm{~mm}$. (G) The effect of Wnt inhibitor on sphere formation ability was evaluated by counting and comparing the total number of spheres. DMSO served as negative control. ${ }^{*} \mathrm{P}<0.05 ;{ }^{*} \mathrm{P}<0.01$.

\section{Supplementary Files}

This is a list of supplementary files associated with this preprint. Click to download.

- SupplementaryTables.docx 